\title{
Circulating miR-20a and miR-26a as Biomarkers in Prostate Cancer
}

\author{
Peyman Mohammadi Torbati ${ }^{1}$, Fatemeh Asadi $^{2}$, Pezhman Fard-Esfahani2*
}

\begin{abstract}
Objective: Circulating microRNAs (miRNAs), present in body fluids, have been considering importance as cancer biomarkers. The primary aim of this study was to assess whether circulatory miR-20a and miR-26a can be used as diagnostic biomarkers in prostate cancer (PCa). Methods: Relative expression miR-20a and miR-26a has been assessed in 40 patients with PCa and 40 non-cancerous volunteer. Sample Collection of patients was performed before and one week after prostatectomy. Total RNA was extracted from serum and miR-20a and miR-26a expressions were quantified by using Real-Time PCR method. Results: miR-20a was significantly up-regulated in pre-operation serum samples of PCa patients compared to the serum samples of non-cancerous controls, however, in post-operation samples no significant differences was showed. miR-26a level was not significantly decreased in pre and post-operation serum samples compared to the serum samples of controls. However, the expression level ratios of both miR-20a and miR-26a were insignificantly decreased when post-operation serum samples compared to pre-operation ones. Conclusion: Decrement of circulating miR-20a and miR-26a in patients after surgery may reflect the tumoral origin of those microRNAs and the results may use for tumor remnant monitoring after prostatectomy.
\end{abstract}

Keywords: Prostate cancer- circulatory microRNAs- miR-20a- miR-26a

Asian Pac J Cancer Prev, 20 (5), 1453-1456

\section{Introduction}

Prostate cancer $(\mathrm{PCa})$ is the second most common tumor after skin cancer among men. It is also the second prominent cause of male cancer death in the USA (Sonn et al., 2005; Qiang et al., 2014). Like most other cancers, biology of PCa has not been completely apparent and understanding accurate molecular basis of the tumor may help in early diagnosis, monitoring and treatment of the patients. Nowadays for screening and diagnosis of $\mathrm{PCa}$, Digital Rectal Examination (DRE) and serum Prostate Specific Antigen (PSA) assay have been used, although none of them are defect free. The benefit of prostate-specific antigen (PSA) screening has been remained controversial (Leidinger et al., 2016). Drawbacks of PSA and DRE are low specificity and sensitivity, respectively, which results in limitation of their diagnostic value (Backer, 1999). Meanwhile, in the early developmental stages of PCa no clinical symptoms may be seen. These issues have led to unrevealed overwhelming need for investigation on new biomarkers which can prevail these weak points and make early diagnosis feasible.

MicroRNAs (miRNAs) are a cluster of non-coding, endogenous and small RNA molecules which have important functions in regulation of various biological processes such as proliferation, differentiation, apoptosis, carcinogenesis and metastasis (Esquela-Kerscher and Slack, 2006; zur Hausen, 2008; Wahid et al., 2010; Saini, 2016). Previous studies suggested miRNAs may play dual roles in cancer biology as oncogenic or tumorsupressor (Esquela-Kerscher and Slack, 2006; Zhang et al., 2007). Existence and stability of miRNAs in body fluids such as serum and plasma samples have been previously reported (Mitchell et al., 2008). Firstly, circulating microRNAs (c-miRNAs) have been described by Lawrie et al. They have also suggested c-miRNAs as diagnostic markers for cancers (Lawrie et al., 2008).

As blood base biomarkers including c-miRNAs are very beneficial in diseases monitoring, recent studies have proposed circulating microRNAs could be used as noninvasive predictor biomarkers of various cancers including PCa (Srivastava et al., 2011; Bryant et al., 2012; McDonald et al., 2018). Many c-miRNAs have been reported as biomarkers of solid tumors (Pritchard et al., 2012). Porkka et al., (2007) identified 51 individual miRNAs which differentially expressed between benign and malignant tumors, among which, 37 showed down-regulation and 14 showed up-regulation in carcinoma samples. In other studies higher levels of miR-20a expression were shown in more dedifferentiated PCa cells (Pesta et al., 2010) and other cancers (Zhang et al., 2018). Ambs et al., (2008) suggested that microRNA 
expression changes with development and progression of prostate cancer and microRNAs regulated some of the cancer-related genes. In the study of Brase et al., (2011) the investigators concluded that miR-375 and miR141 expression is enhanced in prostate cancer samples and their release into the blood is in association with progressed cancer disease. Mahn et al., (2011) have been reported expression pattern of selected oncogenic miRNAs in serum and demonstrated that miR-26a level was able to distinguish $\mathrm{PCa}$ and $\mathrm{BPH}$ patients (sensitive $89 \%$ and $56 \%$ specificity).

We selected miR-20a and miR-26a for analysis based on previous impellent studies which described the importance of these miRNAs in cancers (ParvizHamidi et al., 2018; Xu et al., 2018). For example there are studies expressed that miR-20a regulates cell growth and plays important roles in prostate and other cancers initiation and progression (O'donnell et al., 2005; Pesta et al., 2010; Liu et al., 2012; Xu et al., 2018; Zhang et al., 2018). Shen et al., (2012) represented significant overexpression of miR-20a in plasma from patients with stage $3 \mathrm{PCa}$ tumors compared to stage 2 or below. On the other hand, it is suggested that miR-26a plays significant role in a number of human cancers (Ji et al., 2009; Srivastava et al., 2011; Deng et al., 2013; Gao et al., 2013; Jiang et al., 2014; Shen et al., 2014; Liu et al., 2015; Li et al., 2018). Zhang $\mathrm{J}$ et al, reported non-tumor tissues showed significantly increased miR-26a expression compared to prostate cancer tissues (Zhang et al., 2016). Although there are several studies in this field, more researched are needed for confirmation of reliable biomarkers for prediction of PCa. In current study the primary aim was expression evaluation of miR-20a and miR-26a in serum sample of healthy controls in comparison with PCa patients before and after radical prostatectomy by Quantitative Real-time PCR (qRT-PCR).

\section{Materials and Methods}

\section{Blood Samples}

We collected peripheral blood samples of patients and healthy volunteers ( $n=40$ and $n=40$ respectively) after receiving informed consent. The volunteers had no history of cancer until sample accumulation. Collection was performed before operation and one week post-operation. Samples were centrifuged at 3,000 g for 10 minutes at $4^{\circ} \mathrm{C}$ For isolation of serum. Then Samples stored at $-80^{\circ} \mathrm{C}$ until needed.

\section{RNA isolation and $c D N A$ synthesis:}

Total RNA was extracted by using the miRNeasy Serum/ Plasma Kit (Qiagen) according to the manufacturer's instructions. In order to miRNA normalization 10 picomoles of miRNeasy Serum/Plasma spike-in Control (Qiagen) was added to all serum samples after the addition of QIAzol Lysis Reagent. miRNA molecules were transcribed into cDNA by means of a cDNA Synthesis Kit (miScript II RT QiaGen). Then the resulting cDNA was subjected for qRT- PCR amplification.
The expression of circulating miR-20a and miR-26a in patient and healthy volunteer samples was analyzed by qRT-PCR (Rotor Gene 6000, Corbett Research, Australia). The components in the qRT-PCR reaction for each target were $7 \mu \mathrm{l}$ qPCR Master Mix (All-in-One ${ }^{\mathrm{TM}}$ miRNA qRT-PCR detection kit, QiaGen), $2 \mu$ l of universal reverse-primer sequence in addition to a specific forward primer: miR-20a, TAA AGT GCT TAT AGT GCA GGT AG; miR-26a, TTC AAG TAA TCC AGG ATA GGC T; cel-Mir-39-3p, TCA CCG GGT GTAAAT CAG CTT G. Cycling parameters were set as $95^{\circ} \mathrm{C}$ for $15 \mathrm{~min}, 35$ cycles of $95^{\circ} \mathrm{C}$ for $20 \mathrm{~s}, 54^{\circ} \mathrm{C}$ for $30 \mathrm{~s}, 72^{\circ} \mathrm{C}$ for $30 \mathrm{~s}$.

\section{Statistical analysis}

The analysis of quantitative RT-PCR (qPCR) data was carried out by calculation $2^{-\Delta \Delta \mathrm{Ct}}$ and the defined groups were compared by Student t-test analysis; p-values $<0.05$ considered as significant. The graphs were plotted by Graph Pad Prism 5 software.

\section{Results}

We obtained serum samples from histopathologicallyconfirmed PCa patients $(n=40)$ before and after operation $(n=2 \times 40)$. We also obtained serum samples from 40 non-cancerous men without matching for age. The dataset was obtained after qRT-PCR of miR-20a and miR-26a from pre-operation serum samples, post-operation serum samples and non-cancerous serum samples. The results showed that miR-20a was significantly up-regulated in pre-operation serum samples of PCa patients compared to the serum samples of non-cancerous controls ( $\mathrm{p}$-value $<0.01$ ), however, in post-operation samples showed no significant differences between circular miR-20a compared to same non-cancerous serum controls. Additionally, the results showed significant decrease of miR-20a levels post-operationally compared to the pre-operational samples ( $\mathrm{p}$-value $<0.001$ ). miR-26a

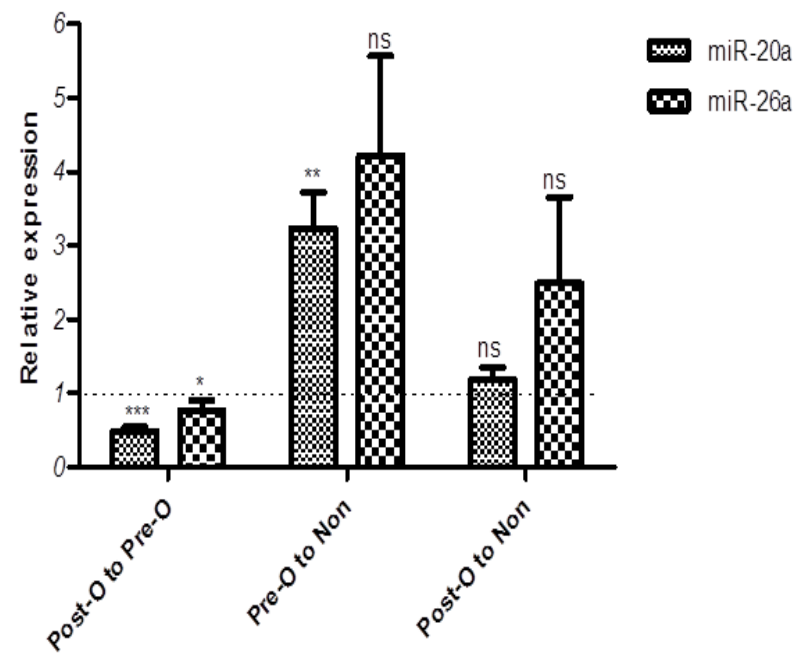

Figure 1. Relative Expression of Circulating miR-20a and miR-26a. Post-O and Pre-O, post-operation and pre-operation serum samples, respectively; ns, nonsignificant; $*$, p-value $<0.05 ; * *$, p-value $<0.01$; ***, p-value $<0.001$. 
level was not significantly decreased in pre and postoperation serum samples compared to the serum samples of health controls. Whereas, in post-operation samples, miR-26a was significantly down-regulated compared to the pre-operational samples ( $\mathrm{p}$-value $<0.05$ ) (Figure 1).

The ratio of expression levels of miR-20a and miR-26a in PCa post-operation serum samples to pre-operation samples were insignificantly altered. This was probably due to surgical removal of tumor, the source of circulating miR-20a and $26 \mathrm{a}$.

\section{Discussion}

High incidence and mortality rate of $\mathrm{PCa}$ lead to it has become a concern in the world (Wolk, 2005). Although there are gradual advances in knowledge about PCa, to improve procedures of prognostic, monitoring and treatment much effort is required. The difference in $\mathrm{PCa}$ incidence in men in various countries can be due to multiple factors such as age, ethnicity, familial and genetic risk, infection, inflammation, androgens, diet, exercise and lifestyle .

Methods which todays are used for evaluation of $\mathrm{PCa}$ are not completely satisfactory so identification of new practical methods are needed (Wang et al., 2008; Suer et al., 2019). As a result, attention has been focused on the miRNAs which frequently dysregulated in cancers. Potential of miRNAs as biomarkers was further amplified by encouraging studies demonstrating that cancer-specific miRNAs are traceable in serum and plasma of patients (Mitchell et al., 2008; Selth et al., 2013; Enokida, 2018). These molecules are remarkably stable in blood and tissues and are resistant to endogenous RNase activity and alterations of temperature and $\mathrm{pH}$, therefore can be used as noninvasive biomarkers. Furthermore there are considerable advantages of applying these molecules for diagnosis of cancers such as ease of measurement, low cost and quick results (Schwarzenbach et al., 2014).

Several studies have been conducted in the field of circulating micro RNAs in PCa. For example Jiang et al., (2014) by monitoring 667 human miRNAs, demonstrated circulating miRNA-375 and miRNA-141 as the most pronounced markers for PCa progression (Brase et al., 2011). In another study, Lin et al., (2014) has identified a panel of circulating microRNAs as potential biomarkers of docetaxel response and overall survival. Recently, Farran et al, investigated a panel of 68 plasma-derived microRNAs (miRNAs) in a cohort study and demonstrate that miR-181a is capable of segregating aggressive and nonaggressive prostate cancer in patients (Farran et al., 2018).

In the present study circulating mir20a and mir26a have been evaluated because in previous studies significance of mentioned miRNAs in initiation of $\mathrm{PCa}$ and distinction between it and BPH have been expressed (Brase et al., 2011; Mahn et al., 2011; Liu et al., 2012; Shen et al., 2012; Zhang et al., 2016).

The results obtained in this study indicated that increasing the level of miR-20a in the serum of patients in comparison with healthy controls can be considered and more tested as a method of early detection of PCa.
Reduction the level of circulating miR-20a and miR-26a in patients after surgery in comparison with before surgery could probably confirm the tumoral origin of them. And analysis of these miRNAs' level may also be considered for tumor remnant monitoring.

It should be noted this study was conducted as pilot and the executives were aware of following weaknesses which seems to be considered in future researches: Small sample size and a few numbers of miRNAs which were analyzed.

\section{Conflict of interest}

Authors claim no conflict of interest statement.

\section{Acknowledgements}

Funding for this research was provided by the Urology and Nephrology Research Center, Shahid Beheshti University of Medical Sciences.

\section{References}

Ambs S, Prueitt RL, Yi M, et al (2008). Genomic profiling of microRNA and messenger RNA reveals deregulated microRNA expression in prostate cancer. Cancer Res, $\mathbf{6 8}$, 6162-70.

Backer H (1999). Prostate cancer screening: exploring the debate. Permanente J, 3, 330-40.

Brase JC, Johannes M, Schlomm T, et al (2011). Circulating miRNAs are correlated with tumor progression in prostate cancer. Int J Cancer, 128, 608-16.

Bryant R, Pawlowski T, Catto J, et al (2012). Changes in circulating microRNA levels associated with prostate cancer. Br J Cancer, 106, 768.

Deng M, Tang H-1, Lu X-h, et al (2013). miR-26a suppresses tumor growth and metastasis by targeting FGF9 in gastric cancer. PLoS One, 8, e72662.

Enokida H (2018). microRNA Analysis in Prostate Cancer. In 'Hormone Therapy and Castration Resistance of Prostate Cancer', Eds Springer, pp 267-91.

Esquela-Kerscher A, Slack FJ (2006). Oncomirs- microRNAs with a role in cancer. Nat Rev Cancer, 6, 259-69.

Farran B, Dyson G, Craig D, et al (2018). A study of circulating microRNAs identifies a new potential biomarker panel to distinguish aggressive prostate cancer. Carcinogenesis, $\mathbf{3 9}$, 556-61.

Gao J, Li L, Wu M, et al (2013). MiR-26a inhibits proliferation and migration of breast cancer through repression of MCL-1. PLoS One, 8, e65138.

Ji J, Shi J, Budhu A, et al (2009). MicroRNA expression, survival, and response to interferon in liver cancer. $N$ Engl J Med, 361, 1437-47.

Jiang D-S, Wang Y-W, Jiang J, et al (2014). MicroRNA-26a involved in Toll-like receptor 9-mediated lung cancer growth and migration. Int J Mol Med, 34, 307-12.

Lawrie CH, Gal S, Dunlop HM, et al (2008). Detection of elevated levels of tumour-associated microRNAs in serum of patients with diffuse large B-cell lymphoma. Br J Haematol, 141, 672-5.

Leidinger P, Hart M, Backes C, et al (2016). Differential bloodbased diagnosis between benign prostatic hyperplasia and prostate cancer: miRNA as source for biomarkers independent of PSA level, Gleason score, or TNM status. Tumor Biol, 37, 10177-85.

Li Y, Li Y, Huang C (2018). Circulating miRNAs Increasing the Risk of Cancer. In 'Cancer and Noncoding RNAs', Eds 
Elsevier, pp 79-94.

Lin H-M, Castillo L, Mahon K, et al (2014). Circulating microRNAs are associated with docetaxel chemotherapy outcome in castration-resistant prostate cancer. Br J Cancer, 110, 2462-71.

Liu D-F, Wu J-T, Wang J-M, et al (2012). MicroRNA expression profile analysis reveals diagnostic biomarker for human prostate cancer. Asian Pac J Cancer Prev, 13, 3313-7.

Liu P, Tang H, Chen B, et al (2015). miR-26a suppresses tumour proliferation and metastasis by targeting metadherin in triple negative breast cancer. Cancer Lett, 357, 384-92.

Mahn R, Heukamp LC, Rogenhofer S, et al (2011). Circulating microRNAs (miRNA) in serum of patients with prostate cancer. Urology, 77, 1265. e9-. e16.

McDonald AC, Vira M, Shen J, et al (2018). Circulating microRNAs in plasma as potential biomarkers for the early detection of prostate cancer. Prostate, 78, 411-8.

Mitchell PS, Parkin RK, Kroh EM, et al (2008). Circulating microRNAs as stable blood-based markers for cancer detection. Proc Natl Acad Sci U S A, 105, 10513-8.

O'donnell KA, Wentzel EA, Zeller KI, et al (2005). c-Mycregulated microRNAs modulate E2F1 expression. Nature, 435, 839-43.

ParvizHamidi M, Haddad G, Ostadrahimi S, et al (2018). Circulating miR-26a and miR-21 as biomarkers for glioblastoma multiform. Biotechnol Appl Biochem, (Page number not assigned).

Pesta M, Klecka J, Kulda V, et al (2010). Importance of miR-20a expression in prostate cancer tissue. Anticancer Res, 30 , 3579-83.

Porkka KP, Pfeiffer MJ, Waltering KK, et al (2007). MicroRNA expression profiling in prostate cancer. Cancer Res, 67, 6130-5.

Pritchard CC, Kroh E, Wood B, et al (2012). Blood cell origin of circulating microRNAs: a cautionary note for cancer biomarker studies. Cancer Prev Res, 5, 492-7.

Qiang XF, Zhang ZW, Liu Q, et al (2014). miR-20a promotes prostate cancer invasion and migration through targeting ABL2. J Cell Biochem, 115, 1269-76.

Saini S (2016). PSA and beyond: alternative prostate cancer biomarkers. Cell Oncol, 39, 97-106.

Schwarzenbach H, Nishida N, Calin GA, et al (2014). Clinical relevance of circulating cell-free microRNAs in cancer. Nat Rev Clin Oncol, 11, 145-56.

Selth L, Townley S, Bert A, et al (2013). Circulating microRNAs predict biochemical recurrence in prostate cancer patients. Br J Cancer, 109, 641-50.

Shen J, Hruby GW, McKiernan JM, et al (2012). Dysregulation of circulating microRNAs and prediction of aggressive prostate cancer. Prostate, 72, 1469-77.

Shen W, Song M, Liu J, et al (2014). MiR-26a promotes ovarian cancer proliferation and tumorigenesis. PLoS One, 9, e86871.

Sonn GA, Aronson W, Litwin M (2005). Impact of diet on prostate cancer: a review. Prostate Cancer Prostatic Dis, 8, 304-10.

Srivastava A, Suy S, Collins SP, et al (2011). Circulating MicroRNA as biomarkers: An update in prostate cancer. Mol Cell Pharmacol, 3, 115.

Suer I, Guzel E, Karatas OF, et al (2019). MicroRNAs as prognostic markers in prostate cancer. Prostate, 79, 265-71.

Wahid F, Shehzad A, Khan T, et al (2010). MicroRNAs: synthesis, mechanism, function, and recent clinical trials. Biochim Biophys Acta-Mol Cell Res, 1803, 1231-43.

Wang P, Guo Y-m, Liu M, et al (2008). A meta-analysis of the accuracy of prostate cancer studies which use magnetic resonance spectroscopy as a diagnostic tool. Korean $J$
Radiol, 9, 432-8.

Wolk A (2005). Diet, lifestyle and risk of prostate cancer. Acta Oncol, 44, 277-81.

$\mathrm{Xu} \mathrm{X}$, Zhu S, Tao Z, et al (2018). High circulating miR-18a, miR-20a, and miR-92a expression correlates with poor prognosis in patients with non-small cell lung cancer. Cancer Med, 7, 21-31.

Zhang B, Pan X, Cobb GP, et al (2007). microRNAs as oncogenes and tumor suppressors. Devel Biol, 302, 1-12.

Zhang J, Liang J, Huang J (2016). Downregulated microRNA-26a modulates prostate cancer cell proliferation and apoptosis by targeting COX-2. Oncol Lett, 12, 3397-402.

Zhang Q, Wang Q, Sun W, et al (2018). Change of circulating and tissue-based miR-20a in human cancers and associated prognostic implication: A systematic review and meta-analysis. BioMed Res Int, 2018, Article ID 6124927, 14 pages.

zur Hausen H (2008). The role of microRNAs in human cancer. Int $J$ Cancer, 122, ix-ix.

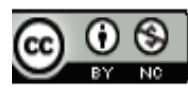

This work is licensed under a Creative Commons AttributionNon Commercial 4.0 International License. 